Appealing to the Base or to the Moveable Middle? Incumbents' Partisan Messaging Before the 2016 U.S. Congressional Elections

Libby Hemphill1 (@libbyh) and Matthew A. Shapiro² ${ }^{1}$ University of Michigan

2 Illinois Institute of Technology 


\section{Main takeaway}

Democrats and Republicans differed in their partisanship:

- Democrats decreased their partisanship, following the "median voter" playbook

- Republicans remained consistent in their messaging, using Twitter to activate and reinforce their base 


\section{Background}

- Median Voter Theorem (Downs 1957)

- Activation and reinforcement (Lazarsfeld, Berelson, and Gaudet 1948)

- Direct (Mitchell, Gottfried, Barthel, \& Shearer, 2016) and indirect (Shapiro and Hemphill 2017) political audiences on Twitter

- Measuring polarization through tweets (Hemphill, Culotta, and Heston 2016) 


\section{Hypotheses}

1. As the election nears, politicians will exhibit lower polarization scores.

2. As the election nears, politicians will exhibit higher polarization scores.

3. Majority party incumbents will exhibit lower polarization scores than minority party incumbents.

4. Candidates in close races will exhibit lower polarization scores. 


\section{Why both higher and lower?}

1. Median voter theorem: reduce partisanship to attract the moveable middle

2. Activate and Reinforce: increase partisanship to get base to the polls

3. Low Congressional approval + unpopular presidential candidate: reduce partisanship to appear less extreme

4. Close race: reduce partisanship to reduce effect of party affiliation 


\section{\#Polar Scores for Measuring Partisanship on Twitter}

1. Collect tweets

2. Identify "framing" or "positioning" hashtags

3. Create binary hashtag vectors for each MOC

4. Run through feature selection algorithms, where hashtags are features

5. Assign signed scores to tags: \#Polar-Hashtag

6. Sum signed tag scores: \#Polar-User 


\section{Methods}

1. Collect tweets from incumbents $(\mathbf{2 5 , 4 8 3}$ tweets from $\mathbf{4 5 8}$ accounts)

2. Calculate \#polar scores for each week from Labor Day to Election Day

3. Predict \#polar scores using individual, party, time, and race measures 


\section{Measures}

\begin{tabular}{|l|l|l|}
\hline Variable & Type & Operationalization \\
\hline abs & outcome & $\begin{array}{l}\text { Absolute value of the average partisanship of the } \\
\text { member of Congress's Twitter feed for week }\end{array}$ \\
\hline handle & predictor & $\begin{array}{l}\text { Twitter handle associated with the member of } \\
\text { Congress's account }\end{array}$ \\
\hline party & predictor & $1=$ Republican; 0 = Democratic \\
\hline week & predictor & Number of the week (1= week beginning Labor Day) \\
\hline margin of & predictor & $\begin{array}{l}\text { Ratio of votes separating the winner and the runner-up } \\
\text { to sum of votes both candidates received }\end{array}$ \\
\hline
\end{tabular}




\section{\#Polar scores over time}

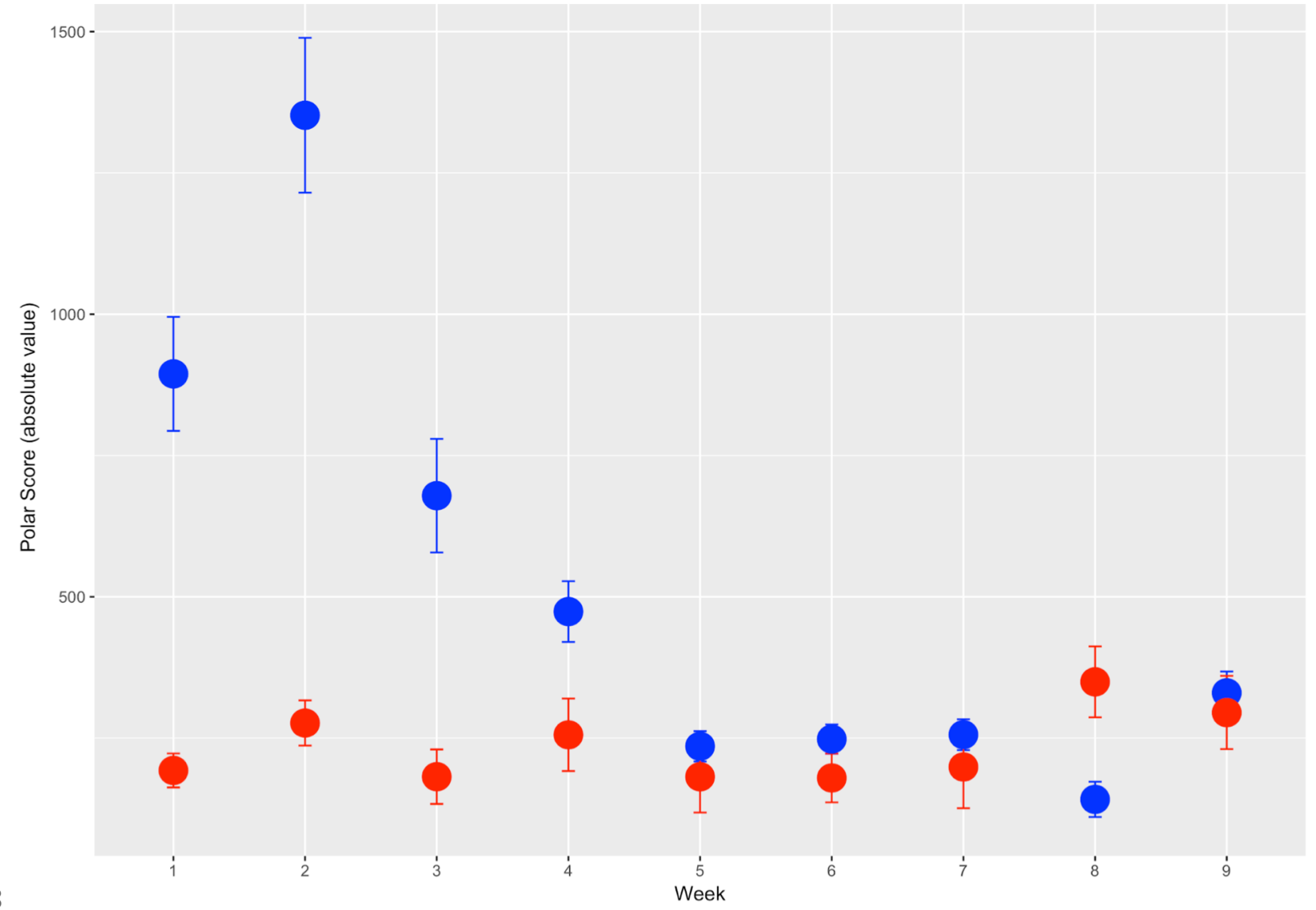




\begin{tabular}{|c|c|c|c|}
\hline & Overall Model & Republicans & Democrats \\
\hline \multicolumn{4}{|l|}{ Fixed Effects } \\
\hline Week & $\begin{array}{c}-117.29^{* * *} \\
(9.32)\end{array}$ & $\begin{array}{c}2.82 \\
(4.40)\end{array}$ & $\begin{array}{c}-110.01^{* * *} \\
(12.99)\end{array}$ \\
\hline Party (Republican) & $\begin{array}{c}\mathbf{- 8 7 8 . 6 0} \\
(95.63)\end{array}$ & & \\
\hline Week* Party & $\begin{array}{c}125.10^{* * *} \\
(12.56)\end{array}$ & & \\
\hline \multicolumn{4}{|l|}{ Random Effects } \\
\hline Handle & 324614 & 148321 & 487028 \\
\hline Handle, week & 779051 & 154608 & 1640135 \\
\hline \multicolumn{4}{|l|}{ Model Fit } \\
\hline AIC & 49875 & 25868 & 23113 \\
\hline
\end{tabular}




\begin{tabular}{|l|c|c|}
\hline & Week alone & Including race margin \\
\hline Fixed Effects & \multicolumn{2}{|c|}{} \\
\hline Week & $-\mathbf{4 7 . 4 4 * * *}$ & $\mathbf{- 1 1 7 . 2 7 ^ { * * * }}$ \\
\hline Party (Republican) & $(9.91)$ & $(9.318)$ \\
\hline Margin & & $-\mathbf{8 7 4 . 0 7 * *}$ \\
& & $(96.155)$ \\
\hline Week * Party & & 0.653 \\
& & $(1.170)$ \\
\hline Model Fit & & $125.077^{* * *}$ \\
\hline AIC & & $(12.563)$ \\
\hline
\end{tabular}




\section{Results}

\section{Hypothesis}

Median Voter: Lower scores

IPP: Higher scores

Unpopular Congress, presidential candidate: Lower scores

Close race: Lower scores

\section{Result}

Supported

Not supported

Supported

Not supported 


\section{Takeaways}

- Republicans and Democrats employed different strategies.

- Republicans - stake a moderate claim and stay there (mostly)

- Democrats - message in line with Congressional action, move to the middle right before the election

- Trump didn't make 2016 unique, at least not on this measure.

- Future work: challengers and campaign accounts 
Supplemental Slides 


\section{Evaluating \#Polar Scores Algorithms}

- Split $D$ into $k$ equal-sized sets $D_{1} \ldots D_{k}$

- For each set

- Construct $D_{\text {train }}=D \backslash D_{k} ; D_{\text {test }}=D_{k}$

- Rank features in Dtrain according to $F$

- Retain the top $m$ features

- Fit a classifier on Dtrain using only the selected $m$ features

- Predict the class assignments for the held-out observations in Dtest 


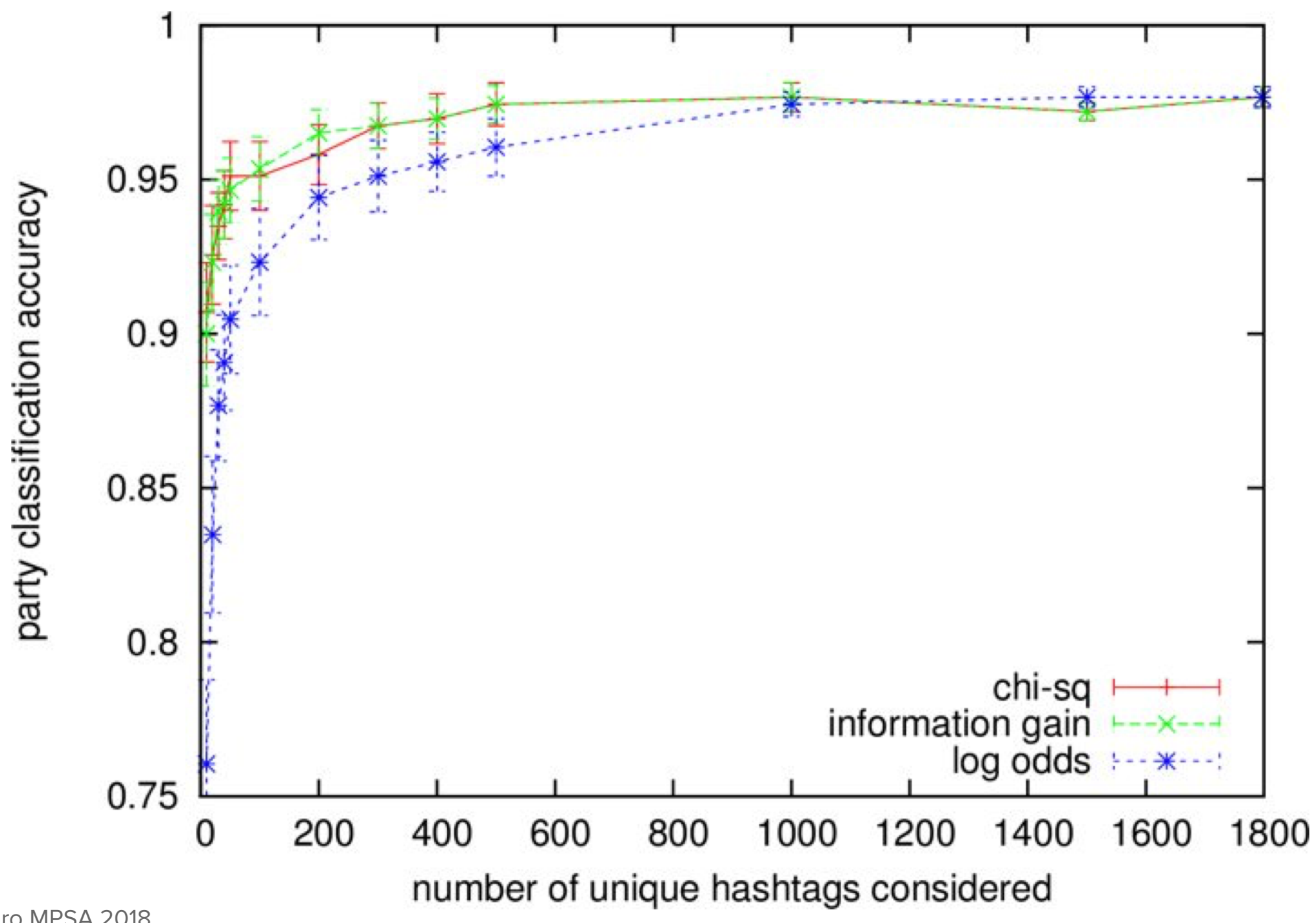

